\title{
Lateral ventricular medulloepithelioma in children: a case report
}

\author{
Hong You", Jiangtao Dong", Jian Xu, Dong Zhao, Qi Liu \\ Department of Neurosurgery, The First Affiliated Hospital, Shihezi University School of Medicine, Shihezi, China \\ \#These authors contributed equally to this work. \\ Correspondence to: Qi Liu. Department of Neurosurgery, The First Affiliated Hospital, Shihezi University School of Medicine, Shihezi, China. \\ Email: liuqi801020@163.com.
}

\begin{abstract}
Medulloepithelioma is an extremely rare highly malignant and rapidly growing tumor that occurs in the central nervous system. There are few reports of medulloepithelioma located in the ventricle. Medulloepithelioma is common in young children and adolescence. Herein, we described an unusual case of vomiting in a 4-year-old male patient with medulloepithelioma, presenting with enlarging head circumference. Because of computed tomography (CT) scan of the head showed signs of brain tumors and hydrocephalus, and enhanced magnetic resonance imaging (MRI) sequence showed increased heterogeneity and honeycomb-like changes on the mass after the administration of a contrast agent, the patient was first diagnosed as choroid plexus papilloma. After undergoing a surgical craniotomy, the patient was diagnosed as medulloepithelioma through pathological examination. We hope that this work will provide more understanding and knowledge of intracranial medulloepithelioma. For medulloepithelioma that occurs in the central nervous system, radiological examination is not sufficient to make a definite diagnosis of the tumor. Pathological examination can confirm the diagnosis of medulloepithelioma and distinguish it from other central system tumors. Surgical resection is a safe and effective method that can prolong the life of patients. However, the prognosis of medulloepithelioma is still poor, and further research is needed to improve the diagnosis and treatment of this rare disease.
\end{abstract}

Keywords: Medulloepithelioma; central nervous system; lateral ventricular

Submitted Aug 20, 2020. Accepted for publication Feb 24, 2021.

doi: $10.21037 / \mathrm{tp}-20-257$

View this article at: http://dx.doi.org/10.21037/tp-20-257

\section{Introduction}

Medulloepithelioma is a rare highly malignant embryonic tumor that originates from primitive pluripotent and neural tube-specific pluripotent stem cells. The peak incidence is between 6 months and 5 years of age $(1,2)$. In 2016, the World Health Organization proposed a new classification for tumors of the central nervous system, and the term "primitive neuroectodermal tumors" has been removed from the dictionary. However, the names of some rare solid tumors are reserved, such as medulloepithelioma. The histological features of medulloepithelioma are the papillary, tubular, or trabecular arrangement of the tumor neuroepithelium, which mimics the embryonic neural tube (3). Medulloepithelioma that occurs in the orbit usually shows a benign clinical course after simple resection, but the prognosis of medulloepithelioma that occurs in the intracranial area is poor, especially when the tumor is not completely removed (4). In this study, we report a 4-yearold child who was diagnosed with choroid plexus papilloma based on the initial head magnetic resonance imaging (MRI) results. The postoperative pathological results confirmed that he had medulloepithelioma. The study of this case will help us to improve our understanding of the diagnosis of medulloepithelioma.

We present the following case in accordance with the CARE reporting checklist (available at http://dx.doi. org/10.21037/tp-20-257).

\section{Case presentation}

A 4-year-old boy was admitted to the pediatrics department of our hospital due to vomiting for 1 week. On physical 

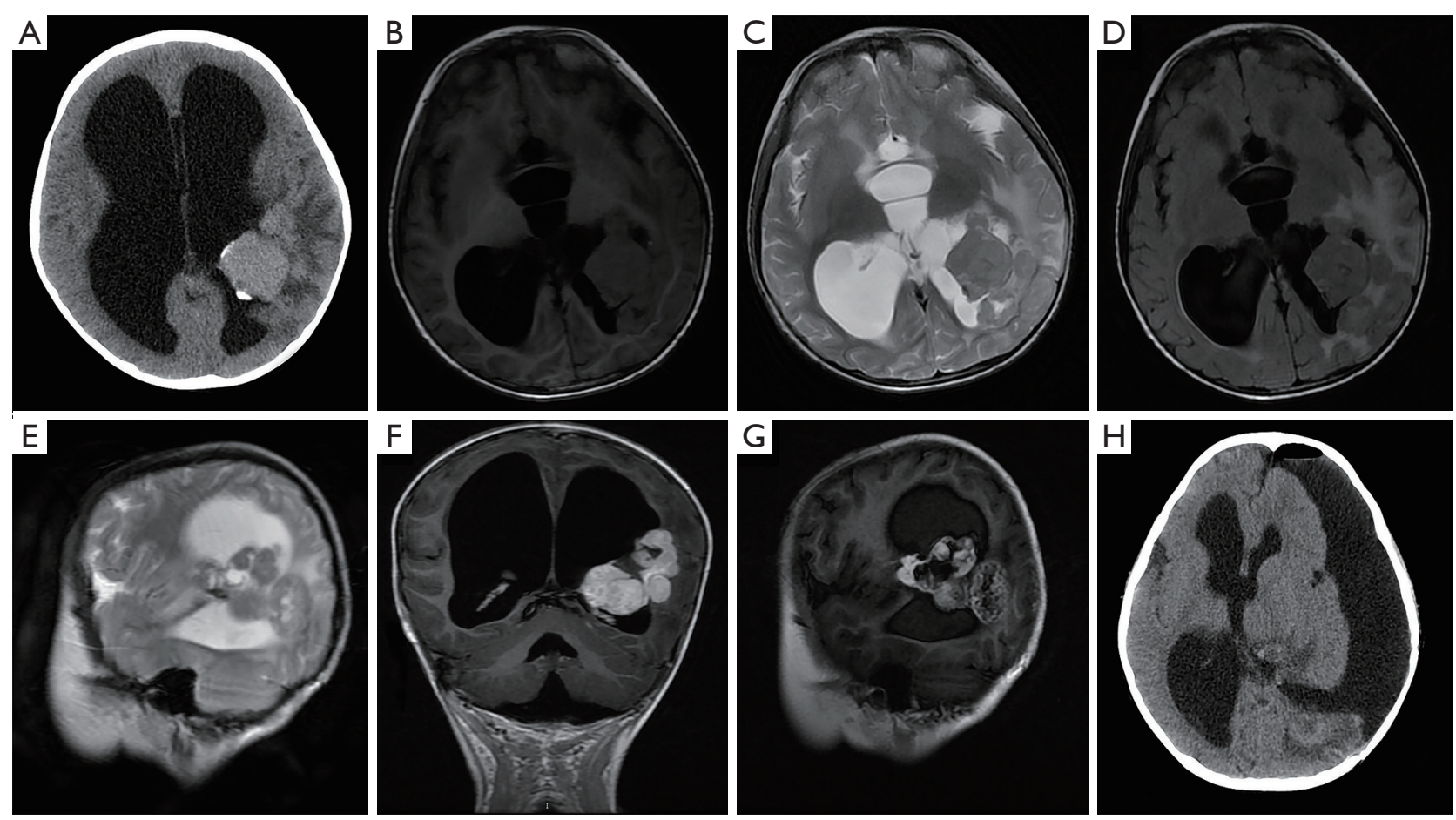

Figure 1 Images of the patient. (A) The patient's preoperative CT showed that the mass in the left lateral ventricle was isodense with enhanced margins and hydrocephalus. (B) Preoperative T1-weighted MRI fluid attenuated inversion recovery (FLAIR) sequence axial view: a mass in the left lateral ventricle. (C) Preoperative T2-weighted MRI axial view: a mass in the left lateral ventricle and surrounding cerebrospinal fluid. (D) Preoperative T2-weighted MRI FLAIR sequence axial position: a mass in the left lateral ventricle and compression of the surrounding brain tissue. (E) The sagittal view of the preoperative T1-weighted MRI showed a mass in the left lateral ventricle, some of which exhibited high-density changes, and the mass was obviously heterogeneous. (F) The coronal view of enhanced T1-weighted MRI showed that the masses in the left lateral ventricle were obviously heterogeneous and accompanied by local heterogeneous low density. (G) The sagittal view of enhanced T1-weighted MRI showed that the local heterogeneity of the mass in the left lateral ventricle was enhanced, accompanied by local heterogeneous low density. (H) The postoperative CT of the patient showed that the lesion was completely removed, and hydrocephalus was still present. CT, computed tomography; MRI, magnetic resonance imaging.

examination, the child had an enlarged head circumference $(53 \mathrm{~cm})$, and the rest were normal. Laboratory examination showed no abnormalities. Subsequently, the child suddenly twitched his limbs with eyes wide open and teeth clenched. A computed tomography (CT) scan of the head showed signs of brain tumors and hydrocephalus. After being transferred to our department, the brain MRI scan sequence showed some cystic heterogeneous masses in the left lateral ventricle and hydrocephalus formation. The enhanced sequence showed the following: the coronal and sagittal images exhibit increased heterogeneity of the mass after the administration of a contrast agent, the enhanced separation is obvious, and "honeycomb-like" changes can be seen locally. The initial MRI diagnosis was choroid plexus papilloma in the triangle of the left lateral ventricle and hydrocephalus (Figure 1).

After explaining the condition and treatment plan to the child's parents, a craniotomy was performed. During the operation, the tumor was irregular in shape, light gray, soft, and cystic. The tumor had a clear boundary from the surrounding brain tissue and was completely removed. The pathological results of rapid freezing during the operation were as follows: tend to medulloblastoma or primitive neuroectodermal tumor. The postoperative CT of the skull showed that the tumor had been completely removed. The histopathological results of the child later showed brain embryonic tumor - medulloepithelioma (WHO grade IV). Later, we found no shed tumor cells in the cerebrospinal fluid. The child's vomiting condition eased in the following days. However, the hydrocephalus in the brain was not 

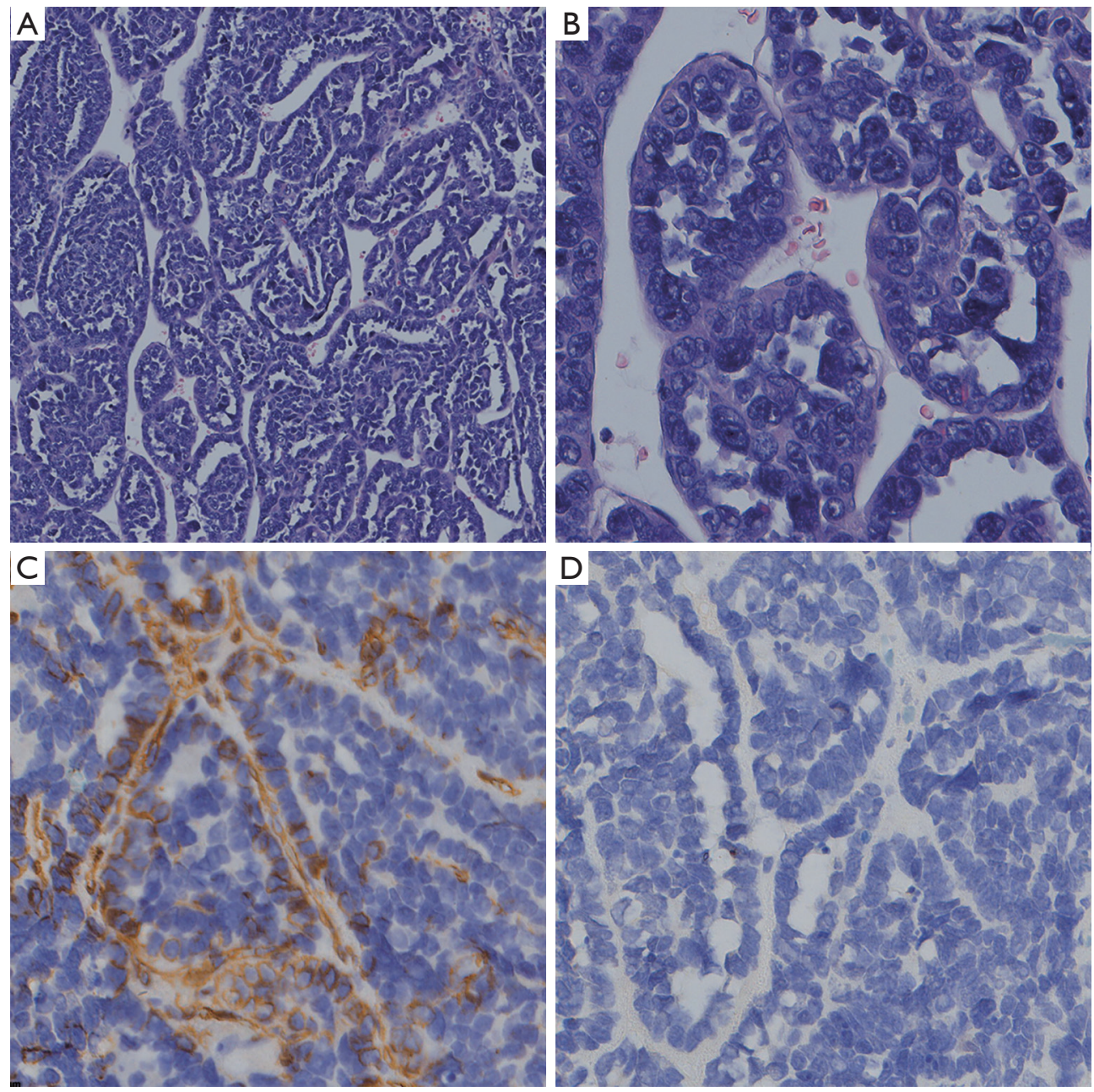

Figure 2 Micrograph of the tumor of the patient. (A) Histological examination showed tumor epithelial cells with adenoid, papillary, cordlike, or nest-like arrangements $(\mathrm{H} \& \mathrm{E}, \times 10)$. (B) Mitotic cells were located on the luminal surface of the pseudostratified tissue $(\mathrm{H} \& \mathrm{E}, \times 40)$. (C) Immunohistochemical analysis revealed partially positive expression of vimentin (IHC, $\times 40$ ). (D) Negative stain for CK7 (IHC, $\times 40$ ). H\&E, hematoxylin-eosin staining; IHC, Immunohistochemistry.

relieved, and we inserted a ventricular-abdominal shunt on the child. Later, the parents of the child refused chemotherapy or radiation therapy. Unfortunately, he was lost to follow-up after 2 months.

Histopathology showed that the tumor cells were singleor multi-layered and exhibited adenoid, papillary, cordlike, or nest-like arrangement. Some small nucleoli were seen, and pathological mitotic figures were easy to observe. Immunohistochemical analysis showed the following: hPL(-), HCG(focus +), OCT-3/4(-), PLAP(-), NKX2.2(-), $\operatorname{AFP}(-), \operatorname{CK} 7(-)$, vimentin(-), and CK20(-) (Figure 2).

All procedures performed in studies involving human participants were in accordance with the ethical standards of the institutional and/or national research committee(s) and with the Helsinki Declaration (as revised in 2013). Written informed consent was obtained from the patient's parents.

\section{Discussion}

Medulloepithelioma located in the central nervous system was first described by Bailey and Cushing in 1926 (5). Medulloepithelioma accounts for $1 \%$ of primary central nervous system tumors and is an extremely rare embryonic tumor (6). This disease usually occurs in young children or adolescence, with a peak incidence of 6 months to 5 years of age. It has no gender difference, and it is rare in adults $(2,4)$. 
Medulloepithelioma is currently considered to be a tumor that originates from primitive pluripotent and neural tubespecific pluripotent stem cells, which can occur anywhere in the entire nerve axis (7). Medulloepithelioma mostly occurs in the cerebral hemisphere, brainstem, cerebellum, or peripheral nerves. The brain is the most common location in the central nervous system, accounting for about $30 \%$. The most common location is usually near the ventricle, followed by the temporal lobe, parietal lobe, occipital lobe, and temporal lobe (in order of frequency) (7). Known for its high degree of malignancy, medulloepithelioma has a poor prognosis. Despite aggressive treatment, the median survival time is still 5 months, and there is no tendency for gender differences (8). Moreover, medulloepithelioma can also occur in the orbit. Contrary to medulloepithelioma located in the central nervous system, a better long-term prognosis can be obtained only by eye removal in cases that occur in the orbit (4). The survival difference of these two tumor entities is related to the molecular diversity of cytogenetics and epigenetics, which indicate that they are different disease entities (9).

Histologically, tumor cells are arranged in tubules, trabeculae, or papillae. Compared with normal cells, tumor cells are larger, round, or polygonal, with less eosinophilic cytoplasm. The tumor cell nuclei are round or oval, with different sizes and deep staining, and the chromatin is granular, with more nucleoli, increased nuclear heterogeneity, and significantly reduced cytoplasm $(2,6)$. Tumor cells may have multiple directions of differentiation, including mesenchymal cells, neurons, astrocytes, ependymal cells, or oligodendrocytes, which can be detected by immunohistochemistry. Tumors usually respond to synaptophysin and waveform protein, and glial fibrillary acidic protein or neuronal characteristic enolase is positively expressed $(2,10)$. Due to the direction of mesenchymal differentiation, teratoma and medulloepithelioma may have histogenetic overlap. Therefore, the diagnostic markers of medulloepithelioma still need to be determined, and the diagnosis of medulloepithelioma depends largely on microscopic observation.

The radiological features of medulloepithelioma are obvious, but not specific. In CT scans, most medulloepitheliomas have low density or are isodense with clear borders (1). According to the typical MRI of this tumor described by Molloy et al., medulloepithelioma is isodense or has low density in T1-weighted imaging and has high density in T2-weighted imaging, and no enhancement occurs after the use of gadolinium-based contrast agents (11).
However, recent studies have shown that more than $95 \%$ of medulloepithelioma exhibit increased density and contrast enhancement after the use of contrast agents, especially in the solid part of the tumor $(1,12,13)$. In the present study, CT showed an isodense change, and the edges appeared to be significantly enhanced. On MRI, medulloepithelioma showed obvious heterogeneous enhancement and uneven distribution. This may be due to the appearance of cystic necrosis in the tumor, which leads to the destruction of the surrounding environment and blood vessels of the tumor and causes uneven local density enhancement.

The specific location in our case adds to the differential diagnosis of other tumors often seen in these locations. In this case, the preoperative MRI revealed an intraventricular tumor with similar characteristics: choroid plexus papilloma. The MRI features of choroid plexus papilloma are currently described as having isodensity or mild low density in T1weighted imaging and heterogeneous and isodense lobular mulberry-like masses in T2-weighted imaging. After injection of gadolinium contrast agent, choroid plexus papilloma showed obvious uniform or uneven enhancement (14). In our case, these features are not different from the MRI findings. The only accurate way to confirm the diagnosis is through histopathological examination. Our pathology results are consistent with those of previous literature (7). The histological characteristics of choroid plexus papilloma are well-differentiated columnar epithelial cells and abundant inner capillaries and almost all positive staining for GFAP and CK7 (15). In this case, the tumor cells were observed under a light microscope in adenoid, papillary, cord-like, or nestlike arrangement. The cells were primitive in shape, with sparse cytoplasm, and a large number of pathological mitotic figures were easily seen. Various differentiated cells could be observed, including neurons and glial components. Therefore, we hypothesized that central nervous system tumors that occur in the lateral ventricles of infants and young children are accompanied by hydrocephalus. Especially when the head MRI shows choroid plexus papilloma, we should highly consider whether it is medulloepithelioma. Of course, the final diagnosis depends on observing the morphology of tumor cells under a light microscope after surgery.

At present, due to the rarity of medulloepithelioma, the best treatment option is currently unclear. The current general treatment plan for medulloepithelioma is to first undergo a gross total resection (GTR) and then to perform adjuvant radiotherapy and chemotherapy. Surgical resection is important for patients with intracranial hypertension and can provide treatment opportunities for adjuvant therapy. 
The degree of infiltration of tumor cells into normal brain tissues indicates that patients have a poor prognosis. The removal of tumor and infiltrating tissues around the tumor can be extended to $1 \mathrm{~cm}$, which can improve the prognosis of patients (13). Therefore, intraoperative frozen section is very important to understand the degree of tumor invasion and determine the scope of tumor resection. The best radiotherapy plan includes 35 Gy for 21 days followed by 20 Gy for 12 days for the posterior fossa, primary tumor bed, and postoperative residual tumor. However, radiation therapy can cause cognitive and developmental disabilities in young children, so children under 3 years of age are not suitable for radiotherapy. Active chemotherapy and autologous bone marrow transplant may be a promising strategy. According to Hayase et al., a patient who underwent a total tumor resection without radiotherapy was treated with high-dose chemotherapy (HDCTX) and autologous peripheral blood stem cell transplantation (AuPBSCT) for at least 3 years, and the patient survived (16). According to Moftakhar et al., the characteristics of longterm survival ( $\geq 3$ years) are as follows: tumor is located on the supratentorial region; cerebrospinal fluid specimens are negative for malignant cells; a GTR is obtained; after resection, patients receive aggressive chemotherapy and radiotherapy, regardless of age and gender $(2,6)$. However, another literature review concluded that the position on the screen is not a favorable prognostic factor, and the age at first diagnosis is related to the improvement of patient prognosis (4).

Studies have indicated that the human telomerase catalytic protein subunit (hTERT) gene has been confirmed in medulloepithelioma. According to Fan et al., the hTERT gene is extensively amplified in medulloepithelioma. At the same time, the correlation analysis with patient survival time shows that hTERT is a useful molecular prognostic marker for medulloepithelioma (17).

The limitations of our case are that we lost the patient to follow-up only 2 months later, and we could not determine the prognosis of the child. We did not confirm whether the hTERT gene was expressed in our case.

For medulloepithelioma that occurs in the central nervous system, radiological examination is not sufficient to make a definite diagnosis of the tumor. Its diagnosis depends on histopathological examination results. Surgical resection is a safe and effective method that can prolong the life of patients. However, despite active adjuvant therapy, the prognosis of medulloepithelioma is still poor, and further research is needed to improve the diagnosis and treatment of this rare disease.

\section{Acknowledgments}

We thank Shinewrite (www.shinewrite.com) for its linguistic assistance during the preparation of this manuscript.

Funding: This study was supported by Shihezi University Research Project [grant number ZZZC201822A].

\section{Footnote}

Reporting Checklist: The authors have completed the CARE reporting checklist. Available at http://dx.doi.org/10.21037/ tp-20-257

Conflicts of Interest: All authors have completed the ICMJE uniform disclosure form (available at http://dx.doi. org/10.21037/tp-20-257). The authors have no conflicts of interest to declare.

Ethical Statement: The authors are accountable for all aspects of the work in ensuring that questions related to the accuracy or integrity of any part of the work are appropriately investigated and resolved. All procedures performed in studies involving human participants were in accordance with the ethical standards of the institutional and/or national research committee(s) and with the Helsinki Declaration (as revised in 2013). Written informed consent was obtained from the patient's parents.

Open Access Statement: This is an Open Access article distributed in accordance with the Creative Commons Attribution-NonCommercial-NoDerivs 4.0 International License (CC BY-NC-ND 4.0), which permits the noncommercial replication and distribution of the article with the strict proviso that no changes or edits are made and the original work is properly cited (including links to both the formal publication through the relevant DOI and the license). See: https://creativecommons.org/licenses/by-nc-nd/4.0/.

\section{References}

1. Kusakabe K, Kohno S, Inoue A, et al. Combined morphological, immunohistochemical and genetic analyses of medulloepithelioma in the posterior cranial fossa. Neuropathology 2018;38:179-84.

2. Moftakhar $\mathrm{P}$, Fan $\mathrm{X}$, Hurvitz $\mathrm{CH}$, et al. Long-term survival in a child with a central nervous system 
medulloepithelioma. J Neurosurg Pediatr 2008;2:339-45.

3. Louis DN, Perry A, Reifenberger G, et al. The 2016 World Health Organization Classification of Tumors of the Central Nervous System: a summary. Acta neuropathologica 2016;131:803-20.

4. Müller K, Zwiener I, Welker H, et al. Curative treatment for central nervous system medulloepithelioma despite residual disease after resection. Report of two cases treated according to the GPHO Protocol HIT 2000 and review of the literature. Strahlenther Onkol 2011;187:757-62.

5. Bailey P, Cushing H. A classification of the tumors of the glioma group on a histo-genetic basis with a correlated study of prognosis. Philadelphia: JB Lippincott 1926;54-6.

6. Oumghar N, Hazmiri FE, El Omrani A, et al. Posterior cerebral fossa medulloepithelioma: report of a case. BMC Clin Pathol 2017;17:23.

7. Ramesh AS, Anita M, Jitender S, et al. Unusual occurrence of supratentorial medulloepithelioma in a young female. J Neurosci Rural Pract 2014;5:261-4.

8. Norris LS, Snodgrass S, Miller DC, et al. Recurrent central nervous system medulloepithelioma: response and outcome following marrow-ablative chemotherapy with stem cell rescue. J Pediatr Hematol Oncol 2005;27:264-6.

9. Korshunov A, Jakobiec FA, Eberhart CG, et al. Comparative integrated molecular analysis of intraocular medulloepitheliomas and central nervous system embryonal tumors with multilayered rosettes confirms that they are distinct nosologic entities. Neuropathology 2015;35:538-44.

Cite this article as: You H, Dong J, Xu J, Zhao D, Liu Q. Lateral ventricular medulloepithelioma in children: a case report. Transl Pediatr 2021;10(4):1020-1025. doi: 10.21037/ tp-20-257
10. Chakrabarti I, Majumdar K, Giri A. Infratentorial medulloepithelioma with divergent differentiation: Possibly a predictor of poor outcome. J Pediatr Neurosci 2012;7:142-5.

11. Molloy PT, Yachnis AT, Rorke LB, et al. Central nervous system medulloepithelioma: a series of eight cases including two arising in the pons. J Neurosurg 1996;84:430-6.

12. Lawandy S, Hariri OR, Miulli DE, et al. Supratentorial primitive neuroectodermal tumor in an adult: a case report and review of the literature. J Med Case Rep 2012;6:361.

13. Matsumoto M, Horiuchi K, Sato T, et al. Cerebral medulloepithelioma with long survival. Neurol Med Chir (Tokyo) 2007;47:428-33.

14. Shi Y, Li X, Chen X, et al. Imaging findings of extraventricular choroid plexus papillomas: A study of 10 cases. Oncol Lett 2017;13:1479-85.

15. Gyure KA, Morrison AL. Cytokeratin 7 and 20 expression in choroid plexus tumors: utility in differentiating these neoplasms from metastatic carcinomas. Mod Pathol 2000;13:638-43.

16. Hayase T, Morimoto A, Kawahara Y, et al. An Infant With Medulloepithelioma Successfully Treated by Highdose Chemotherapy Followed by Autologous Peripheral Blood Stem Cell Transplantation Without Radiotherapy. J Pediatr Hematol Oncol 2015;37:e394-8.

17. Fan X, Wang Y, Kratz J, et al. hTERT gene amplification and increased mRNA expression in central nervous system embryonal tumors. Am J Pathol 2003;162:1763-9. 